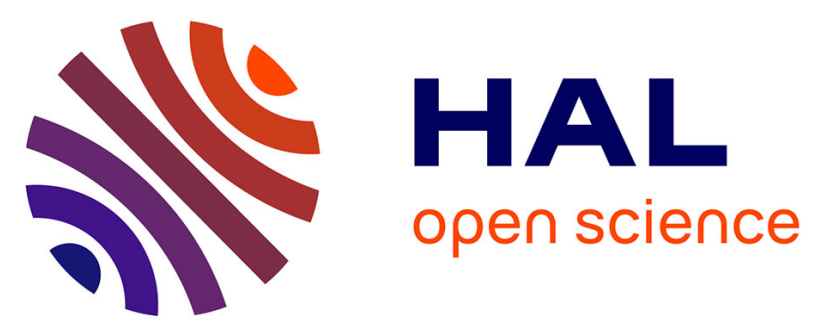

\title{
Factor Analysis of Dynamic Sequence with Spatial Prior for 2D Cardiac Spect Sequences Analysis
}

\author{
Marc Filippi, Michel Desvignes, Eric Moisan, Catherine Ghezzi, Pascale
}

Perret, Daniel Fagret

\section{- To cite this version:}

Marc Filippi, Michel Desvignes, Eric Moisan, Catherine Ghezzi, Pascale Perret, et al.. Factor Analysis of Dynamic Sequence with Spatial Prior for 2D Cardiac Spect Sequences Analysis. ACIVS 2016 International Conference on Advanced Concepts for Intelligent Vision Systems, Oct 2016, Lecce, Italy. pp.228-237, 10.1007/978-3-319-48680-2_21 . hal-01698754

\section{HAL Id: hal-01698754 \\ https://hal.univ-grenoble-alpes.fr/hal-01698754}

Submitted on 1 Feb 2018

HAL is a multi-disciplinary open access archive for the deposit and dissemination of scientific research documents, whether they are published or not. The documents may come from teaching and research institutions in France or abroad, or from public or private research centers.
L'archive ouverte pluridisciplinaire HAL, est destinée au dépôt et à la diffusion de documents scientifiques de niveau recherche, publiés ou non, émanant des établissements d'enseignement et de recherche français ou étrangers, des laboratoires publics ou privés. 


\title{
Factor Analysis of Dynamic Sequence with Spatial Prior for 2D Cardiac Spect Sequences Analysis
}

\author{
M. Filippi, M. Desvignes, E. Moisan ${ }^{1}$, C. Ghezzi, P. Perret, D. Fagret ${ }^{2}$ \\ 1 University Grenoble-Alpes, GIPSA-LAB \\ 2 University Grenoble-Alpes, INSERM1039
}

\begin{abstract}
Unmixing is often a necessary step to analyze 2D SPECT image sequence. However, factor analysis of dynamic sequences (FADS), the commonly used method for unmixing SPECT sequences, suffers from non-uniqueness issue. Optimization-based methods were developed to overcome this issue. These methods are effective but need improvement when the mixing is important or with very low SNR. In this paper, a new objective function using soft spatial prior knowledge is developed. Comparison with previous methods, efficiency and robustness to the choice of priors are illustrated with tests on synthetic dataset. Results on 2D SPECT sequences with high level of noise are also presented and compared.
\end{abstract}

Keywords: Source Separation, SPECT, Factor Analysis, Spatial Priors, Penalized Least Squares.

\section{Introduction}

Insulin resistance is the desensitization of cells to insulin. It can lead to type 2 diabete and cardiovascular disease. Knowledge of the insulin resistance index is of great interest in order to prevent these diseases. However, actual methods are rather invasive and can't be used in clinical routines. A new and easier method has been proposed [1]. It uses a tracer of glucose transport (6-DIG), injected to a patient. The activity of the tracer is dynamically acquired with a gamma camera which produces a 2D SPECT images sequence. Because of the collimator, a very few number of radiations are counted and these images suffer from a high Poisson noise with a very low Signal-to-Noise Ratio (Figure 1).

To compute the insulin resistance index [1], the dynamic activity of the tracer in the myocardium, and in the left and right ventricles (LV and RV) have to be determined. As images are the 2D projections of the 3D organs, spatial overlaps are present in these 2D images, particularly in the cardiac area between the myocardium and the ventricles. Then, to obtain the pure time-activity curve (TAC) of each organ, methods based on region of interest (ROI) give poor results. Because of the spatial overlaps, the activity on these ROIs is composed of a 


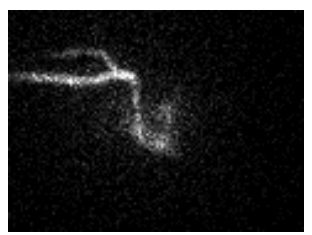

(a)

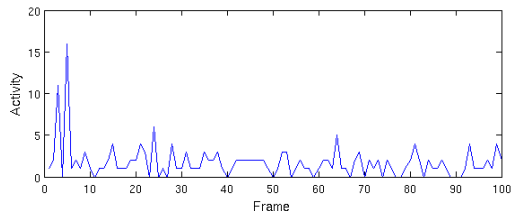

(b)

Fig. 1. 6-DIG SPECT dataset (a) 3rd image of a sequence - Tracer reaching right ventricle (b) Activity of a pixel located on the right ventricle on the first 100 frames.

mixture of pure organs TAC. Therefore, the tracer activity in each physiological compartments have to be unmixed in order to be further analyzed.

It is a common issue in medical imaging, particularly in scintigraphy. Data are assumed to be a mixing of physiological compartments activity and are represented with a linear mixing model (1). Each compartment has its own temporal signature (factor or TAC) and its own spatial signature (factor image).

$$
Y=A \times F+\epsilon
$$

The dynamic data are represented in the $N \times P$ matrix $Y$, where $N$ is the number of pixels (vectorized) and $P$ is the number of images. The $N \times K$ matrix $A$ contains the factor images and the $K \times P$ matrix $F$ contains the factors, where $K$ is the number of physiological compartments. The activity in each pixel is then a linear combination of several factors $F$. The coefficients of this linear combination are contained in the matrix $A$. The matrix $\epsilon$ of size $N \times P$ contains the noise.

The matrix $A$ represents the quantity of each organ on each pixel, and the matrix $F$ represents the number of gamma photons detected on a pixel during a short time span. Then coefficients of matrix $A$ and $F$ must be non-negative. Two classes of methods [2] have been typically used to find $A$ and $F$ with non-negative coefficients by solving equation (1): spectral-type methods [3] and Factor Analysis of Dynamic Structures (FADS) methods [4].

In spectral methods, $F$ is a combination of chosen basis functions such as splines. In general, these methods produce too smooth solutions.

FADS, introduced by Barber [5], shares the same foundation than some Independent Component Analysis (ICA) or Non-Negative Matrix Factorization methods, widely used in hyperspectral unmixing $[6,7,8,9,10]$. FADS has a great geometrical interpretation and proceeds in two steps, an orthogonal analysis followed by an oblique analysis. The first step is used to reduce noise and project data into a low dimension space. The second step uses oblique rotation of factors in order to find factors and factor images with positive coefficients.

FADS has been improved and adapted to scintigraphy [11]. In order to face high Poisson noise an optimal metric has been found [12], and a regularized factor analysis has been proposed [13]. However finding $A$ and $F$ in equation (1) 
with positive constraints is an ill-posed problem, the solution is not mathematically unique, and this leads to high remaining mixture in factors obtained. Prior knowledge on the data has to be added in order to perform the right unmixing and obtain a solution close to the desired one.

Temporal prior knowledge have already been used in [14] and spatial prior knowledge in [15], [16], both with FADS, but these methods fail with very low SNR and high compartments mixing.

In order to overcome non-uniqueness issue of FADS, a penalized least squares objective function has been proposed in [17]. This optimization-based method gives good results when the mixing in the data is low, but results can be improved when the mixing is higher, because solutions with overlaps are penalized in the objective function.

We propose here to exploit soft spatial prior knowledge on the data to improve unmixing in an optimization-based method, by constructing a new objective function. Our method is described in section 2, algorithm and implementation details are given in section 3 and results are shown in section 4 .

\section{Methods}

We propose a new objective function to minimise defined as:

$$
f=f_{L S}(\hat{A}, \hat{F})+a \times f_{U N I}(\hat{A})+b \times f_{P R I O R}(\hat{A})
$$

and under the following constraints :

$$
\forall(i, t, k) \hat{A}_{i, k} \geq 0 \quad \text { and } \quad \hat{F}_{k, t} \geq 0
$$

where $a$ and $b$ are penalty constants. $f_{L S}$ corresponds to the least squares objective. This term measures the distance between the data and the factor model, and ensures data fidelity. This function is not convex in $(\hat{A}, \hat{F})$ but is convex in both $\hat{A}$ and $\hat{F}$, so that we can use an alternate minimization gradient method.

$$
f_{L S}(\hat{A}, \hat{F})=\sum_{i=1}^{N} \sum_{t=1}^{P}\left(Y_{i, t}-\sum_{k=1}^{K} \hat{A}_{i, k} \times \hat{F}_{k, t}\right)^{2}
$$

$f_{U N I}$ penalizes solutions with high correlation between factor images [17] to reduce amount of mixing and favors the separation of factor images with no intersections. It can be seen as a sparsity promoting criterion.

$$
f_{U N I}(\hat{A})=\sum_{k=1}^{K} \sum_{h=k+1}^{P} \sum_{i=1}^{N} \frac{\hat{A}_{i, k}}{\sqrt{\sum_{j=1}^{N} \hat{A}_{j, k}^{2}}} \frac{\hat{A}_{i, h}}{\sqrt{\sum_{j=1}^{N} \hat{A}_{j, h}^{2}}}
$$

$f_{P R I O R}$ is a new penalization term. This criterion ensures fidelity to spatial prior knowledge. The idea behind this criterion is to penalize solutions whose compartments are too far from their expected locations. In Cardiac SPECT 
exams, the ventricles can be easily coarsely located. So, the new term exploits this information to obtain a solution which respect the expected positions of the ventricles.

$$
f_{P R I O R}(\hat{A})=\sum_{k=1}^{K} \sum_{i=1}^{N} \operatorname{dist}_{W_{k}}(i)^{2} \times \hat{A}_{i, k}^{2}
$$

$W_{K}$ is a patch containing a set of pixels where the $k$ th physiological compartment is likely to be located. This patch represents prior knowledge and is the coarse spatial location of the $k^{t h}$ physiological compartment. dist $_{W_{k}}(i)$ is the Euclidean distance between the $i^{t h}$ pixel and the $k^{t h}$ patch (i.e to the closest pixel in the patch). This criterion has the advantage to be convex and computation is fast. Prior knowledge contained in this criterion is also very soft. In most of scintigraphic studies, spatial positions of physiological compartments are easily available thanks to the perfusion. This criterion ensures fidelity with prior knowledge by penalizing factor images far from their expected position. This fidelity is essential in hard unmixing problem to obtain physically meaningful solutions, and overcome non-uniqueness issue.

\section{Algorithm and Implementation details}

Before minimizing the objective function, the number of physiological compartments $K$, the patches $W$ and an initialization of $\hat{A}$ and $\hat{F}$ had to be chosen.

$K$ can be chosen manually with prior knowledge on data, or can be computed with an analysis of singular values given by a singular value decomposition applied on $Y$.

Spatial prior knowledge or patches $W$ can be chosen manually, or automatically with a first segmentation method. In our problem, a first factor analysis gives a first coarse location of LV and RV. Factor images are thresholded and skelotonization creates patches $W_{k}$ containing spatial prior knowledge. When no spatial prior knowledge on a physiological compartment exists, the associated patch contains all the pixels because there are no constraints. Matrix of factor images $\hat{A}$ is initialized by a constant. $\hat{F}$ is initialized with a mean on appropriate ROI for each factor. These ROI can be those used to build the patches $W_{k}$. For the purpose of speeding-up the optimization process, $\hat{F}$ was multiplied by a constant to have the same order of magnitude on $Y$ and $\hat{A} \times \hat{F}$.

As $f_{L S}$ is convex in $\hat{A}$ and convex in $\hat{F}$, but not in $(\hat{A}, \hat{F})$, an alternating minimization method was used. Depending on the alternation, a gradient of the objective function $f$ is calculated analytically according to $\hat{A}$ or $\hat{F}$.

For each alternation, a nonlinear conjugate gradient method is used, with Polak-Ribière method to choose descent direction. The minimum of the objective function in this direction is computed thanks to the Brent method. In order to speed-up the algorithm, the number of iterations for each alternation is limited to 8 and the alternation is stopped if the relative change in the objective function in one iteration is less than $10^{-6}$. 
To satisfy the positivity constraints in (3), the negative coefficients of $A$ and $F$ are forced to zero after each iteration. Each factor image is also re-scaled in order to have their maximum to one after each iteration. Each factors are re-scaled by the reciprocal, in order for (1) to hold.

The optimization process is stopped when the relative change in the objective function between two groups of two alternations is less than $10^{-6}$. We have no proofs of convergence, but in all our tests, the algorithm has always converged in less than 500 alternations.

\section{Results}

\subsection{Synthetic Data}

First, this method is tested on synthetic data set inspired from [17] and representing cardiac area. Dataset is a sequence of 400 images of 30-by-30 pixels. Three physiological compartments are present : right ventricle (RV), left ventricle (LV) and myocardium. Some overlaps are added, in order to have myocardium softly present in the right and left ventricles, as in SPECT sequences.

Poisson noise is added to this data set, with different SNR.

Patches $W_{k}$ are chosen manually, to show robustness of the method to the choice of the prior. The robustness is shown in this example in three ways. Firstly, no prior is selected for the myocardium. Secondly, instead of taking all pixels of the organ (which lead to better results), prior for right and left ventricles are a very thin skeleton of their respective organ. Thirdly, these skeletons are slightly shifted and not exactly located at their right places. These priors are shown on Figure 2. Penalty constants $a$ and $b$ are chosen empirically, a variation of $30 \%$ of these constants does not change significantly the results.

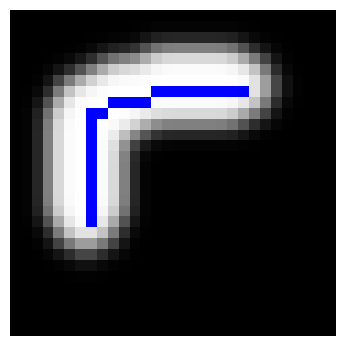

(a)

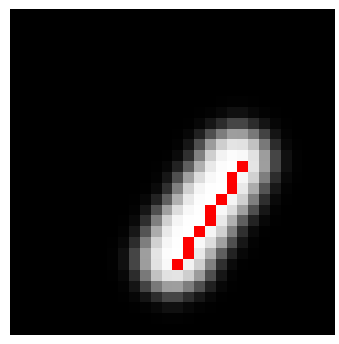

(b)

Fig. 2. Prior used for synthetic data set. (a) Right ventricle in white, and its prior in blue. (b) Left ventricle in white and its prior in red.

Our algorithm $F_{\text {Global }}$ is compared to the optimization-based method $F_{P L S}$ [17] and the regularized FADS method $F_{R E G}[13] . F_{R E G}$ is the method previously 
used for the 6-DIG SPECT data. To compare these algorithms, the errors $A_{\text {error }}$ and $F_{\text {error }}$ are computed. Noise was measured with the formula in (9).

$$
\begin{gathered}
A_{\text {error }}=\frac{\sum_{i=1}^{N} \sum_{k=1}^{K}\left|A_{i, k}-\hat{A}_{i, k}\right|}{\sum_{i=1}^{N} \sum_{k=1}^{K} A_{i, k}} \\
F_{\text {error }}=\frac{\sum_{k=1}^{K} \sum_{t=1}^{P}\left|F_{k, t}-\hat{F}_{k, t}\right|}{\sum_{k=1}^{K} \sum_{t=1}^{P} F_{k, t}} \\
S N R=20 * \log \left(\frac{\sum_{i=1}^{N} \sum_{t=1}^{P}\left|Y_{i, t}\right|^{2}}{\sum_{i=1}^{N} \sum_{t=1}^{P}\left|\epsilon_{i, t}\right|^{2}}\right)
\end{gathered}
$$

Results on synthetic data with different noise levels are detailed in Table I. For $F_{P L S}$ and $F_{\text {Global }}$, penalty constants were first chosen adequately for each noise level. Examples of factors and image factors obtained with synthetic data with a $S N R$ of 10 are shown respectively in Figure 3 and 4 , with $a=150000$ for $F_{P L S}$ and $a=80000$ and $b=300$ for $F_{\text {Global }}$.)

\begin{tabular}{|c|c|c|c|c|}
\hline$S N R$ & & $F_{R E G}$ & $F_{P L S}$ & $F_{\text {Global }}$ \\
\hline \multirow{2}{*}{6} & $A_{\text {error }}$ & 0.298 & 0.225 & 0.169 \\
& $F_{\text {error }}$ & 0.254 & 0.120 & 0.112 \\
\hline \multirow{2}{*}{8} & $A_{\text {error }}$ & 0.237 & 0.172 & 0.135 \\
& $F_{\text {error }}$ & 0.166 & 0.085 & 0.077 \\
\hline \multirow{2}{*}{10} & $A_{\text {error }}$ & 0.206 & 0.142 & 0.109 \\
& $F_{\text {error }}$ & 0.140 & 0.068 & 0.059 \\
\hline \multirow{2}{*}{12} & $A_{\text {error }}$ & 0.184 & 0.110 & 0.088 \\
& $F_{\text {error }}$ & 0.109 & 0.055 & 0.049 \\
\hline
\end{tabular}

Table 1. Comparison of measures $A_{\text {error }}$ and $F_{\text {error }}$, with different methods and noise levels. Each measure is a mean of 20 tests.

Table 1 shows that unmixing is better performed by optimization-based methods $\left(F_{P L S}\right.$ and $\left.F_{G l o b a l}\right)$ than FADS method $\left(F_{R E G}\right)$, for every noise levels and for both estimation of factors and factor images.

Even if the added priors are very soft (just a skeleton) and not perfectly located, the new criterion $f_{P R I O R}$ greatly improves the unmixing, especially on factor images. This new criterion penalizes the factor image corresponding to LV when having high coefficients in the RV area, because the distance to the prior is high. Furthermore, this criterion doesn't penalize myocardium factor image and this myocardium factor image has positive coefficients on the LV or RV area because there is really a superimposition of these organs on the 2D images. At the contrary, the $f_{U N I}$ criterion penalizes overlaps between factor images and can't cope with this problem, and so myocardium is underestimated on the ventricles area with $F_{P L S}$. The improvement on factor image estimation with $F_{\text {Global }}$ leads to a better estimation of factor curves (Figure 4). 

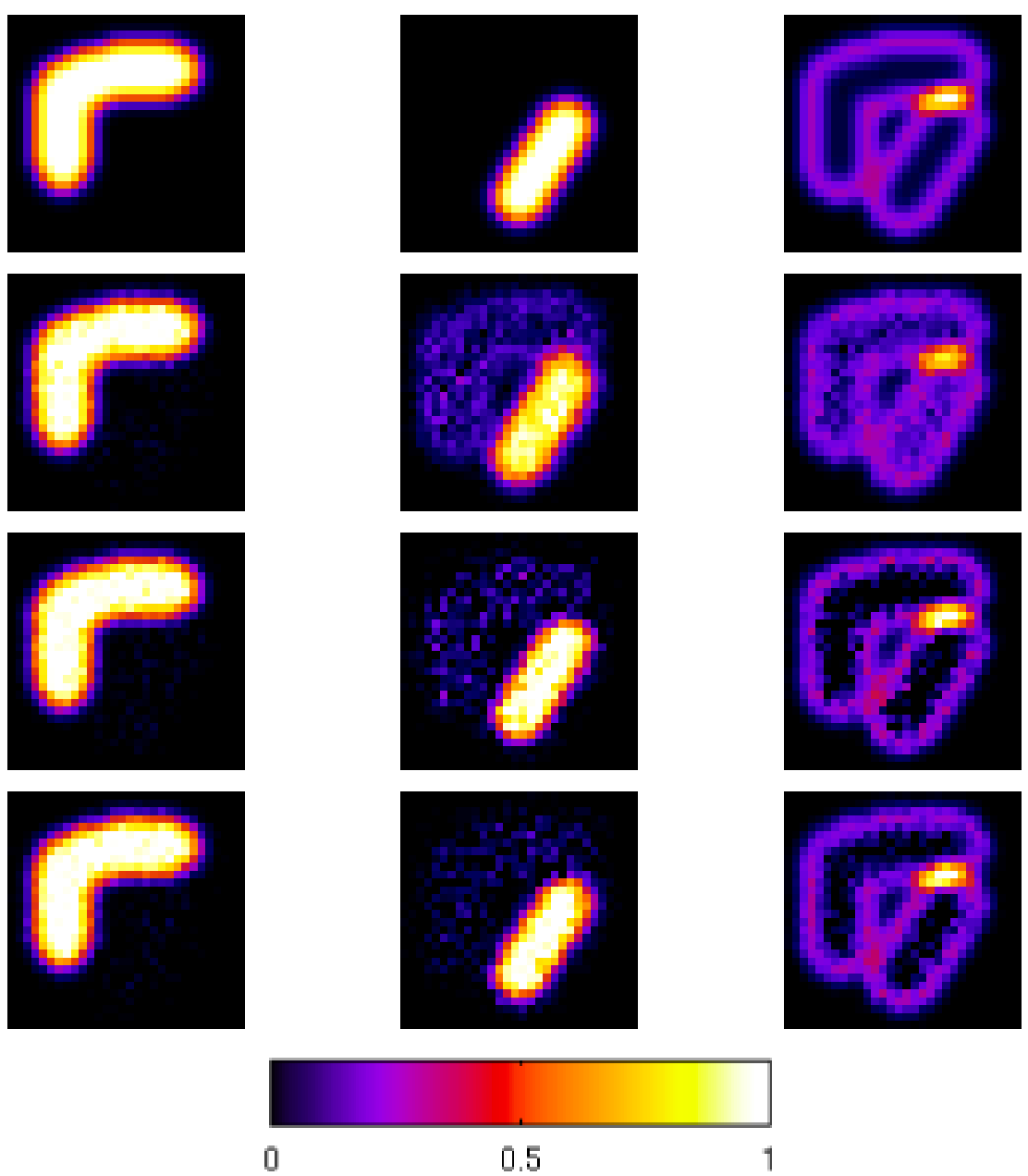

Fig. 3. Comparison of algorithms on synthetic data with a $S N R$ of 10 . First row correspond to ground truth. Row 2, 3 and 4 correspond to factor images obtained respectively with $F_{R E G}, F_{P L S}, F_{\text {Global }}$. Column 1,2 and 3 correspond respectively to RV, LV and myocardium.

\section{$4.2 \quad 6$-DIG SPECT sequence}

The second data set is a 6-DIG SPECT sequence of a patient (see Figure 1), and contains a sequence of 450 images of 128-by-128 pixels. A zoom is performed in order to focus on the cardiac area. Priors chosen are illustrated in Figure 5 and factor images obtained are in Figure 6.

Method $f_{P L S}$ does not have a coherent factor image for the myocardium, this is due to the dimness of the myocardium signal. With $f_{\text {Global }}$, the myocardium is 


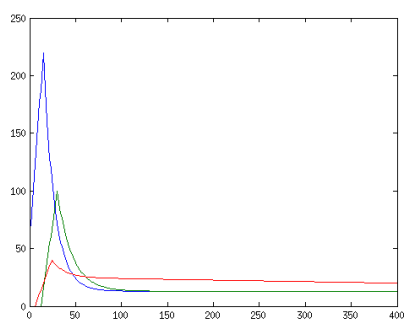

(a)

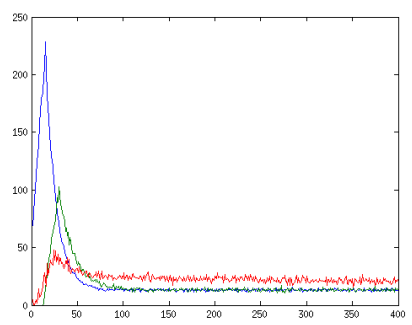

(b)

Fig. 4. Comparison of factors obtained with $F_{\text {Global }}$ on synthetic data with a $S N R$ of 10. (a) Ground Truth (b) Factor obtained with $F_{\text {Global }}$. The blue curves correspond to $\mathrm{RV}$, the red curves to Myocardium, and the green curves to LV.

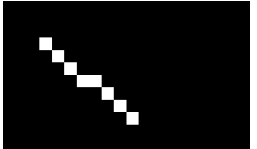

(a)

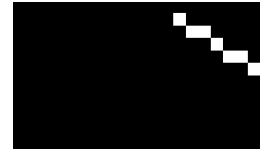

(b)

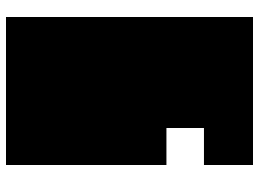

(c)

Fig. 5. Prior used for 6-DIG SPECT data set. Set of pixel $W_{k}$ is represented in white. (a) Prior of RV. (b) Prior of LV. (c) Prior of myocardium.
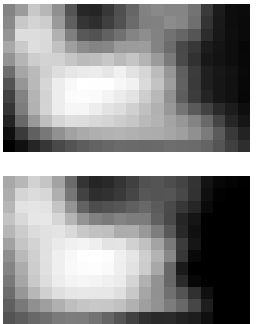
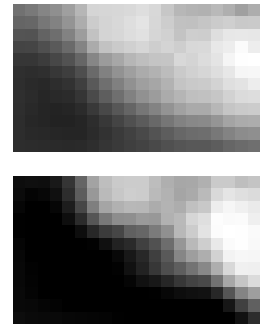
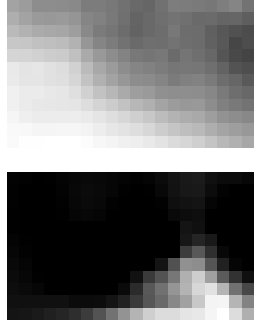

Fig. 6. Factor images obtained when unmixing of 6-DIG dataset. First row : with $f_{P L S}$. Second row : with $f_{\text {Global }}$. Columns 1,2 and 3 represent respectively RV, LV and myocardium.

realistic thanks to the prior located on the cardiac apex, and has been validated by a cardiologist.

\section{Conclusion}

In this paper, we have presented a new spatial prior that can be defined coarsely to improve dynamic sequence analysis. This prior is a convex term and is embedded in an objective function to minimize by an alternate gradient method. 
This prior can be automatically defined because the method is robust to the location and shape of the prior. Tests on synthetic and SPECT dataset show the efficiency and the potential of this method. Future works include tests on larger database and quantitative analysis of the robustness.

\section{References}

[1] Pascale Perret, Lotfi Slimani, Arnaud Briat, Danièle Villemain, Serge Halimi, Jacques Demongeot, Daniel Fagret, and Catherine Ghezzi, "Assessment of insulin resistance in fructose-fed rats with 125i-6-deoxy-6-iodo-d-glucose, a new tracer of glucose transport," European journal of nuclear medicine and molecular imaging, vol. 34, no. 5, pp. 734-744, 2007.

[2] Grant T Gullberg, Bryan W Reutter, Arkadiusz Sitek, Jonathan S Maltz, and Thomas F Budinger, "Dynamic single photon emission computed tomographybasic principles and cardiac applications," Physics in medicine and biology, vol. 55, no. 20, pp. R111, 2010.

[3] Bryan W Reutter, Grant T Gullberg, Rostyslav Boutchko, Karthikayan Balakrishnan, Elias H Botvinick, and Ronald H Huesman, "Fully 4-d dynamic cardiac spect image reconstruction using spatiotemporal b-spline voxelization," in $\mathrm{Nu}$ clear Science Symposium Conference Record, 200\%. NSS'O\%. IEEE. IEEE, 2007, vol. 6, pp. 4217-4221.

[4] Jicun Hu, Rostyslav Boutchko, Arkadiusz Sitek, Bryan W Reutter, Ronald H Huesman, and Grant T Gullberg, "Dynamic molecular imaging of cardiac innervation using a dual head pinhole spect system," Lawrence Berkeley National Laboratory, 2008.

[5] DC Barber, "The use of principal components in the quantitative analysis of gamma camera dynamic studies," Physics in medicine and biology, vol. 25, no. 2, pp. 283, 1980.

[6] Saïd Moussaoui, Hafrun Hauksdottir, Frédéric Schmidt, Christian Jutten, Jocelyn Chanussot, David Brie, Sylvain Douté, and Jon Atli Benediktsson, "On the decomposition of mars hyperspectral data by ica and bayesian positive source separation," Neurocomputing, vol. 71, no. 10, pp. 2194-2208, 2008.

[7] Nicolas Dobigeon, Saïd Moussaoui, Jean-Yves Tourneret, and Cédric Carteret, "Bayesian separation of spectral sources under non-negativity and full additivity constraints," Signal Processing, vol. 89, no. 12, pp. 2657-2669, 2009.

[8] Sen Jia and Yuntao Qian, "Constrained nonnegative matrix factorization for hyperspectral unmixing," Geoscience and Remote Sensing, IEEE Transactions on, vol. 47, no. 1, pp. 161-173, 2009.

[9] Alexis Huck, Mireille Guillaume, and Jacques Blanc-Talon, "Minimum dispersion constrained nonnegative matrix factorization to unmix hyperspectral data," Geoscience and Remote Sensing, IEEE Transactions on, vol. 48, no. 6, pp. 2590-2602, 2010.

[10] José M Bioucas-Dias, Antonio Plaza, Nicolas Dobigeon, Mario Parente, Qian Du, Paul Gader, and Jocelyn Chanussot, "Hyperspectral unmixing overview: Geometrical, statistical, and sparse regression-based approaches," Selected Topics in Applied Earth Observations and Remote Sensing, IEEE Journal of, vol. 5, no. 2, pp. 354-379, 2012.

[11] R Di Paola, JP Bazin, F Aubry, A Aurengo, F Cavailloles, JY Herry, and E Kahn, "Handling of dynamic sequences in nuclear medicine," Nuclear Science, IEEE Transactions on, vol. 29, no. 4, pp. 1310-1321, 1982. 
[12] H Benali, I Buvat, F Frouin, JP Bazin, and R Di Paola, "A statistical model for the determination of the optimal metric in factor analysis of medical image sequences (famis)," Physics in medicine and biology, vol. 38, no. 8, pp. 1065, 1993.

[13] F Frouin, A De Cesare, Y Bouchareb, A Todd-Pokropek, and A Herment, "Spatial regularization applied to factor analysis of medical image sequences (famis)," Physics in medicine and biology, vol. 44, no. 9, pp. 2289, 1999.

[14] J Buvat, H Benali, F Frouin, JP Basin, and R Di Paola, "Target apex-seeking in factor analysis of medical image sequences," Physics in medicine and biology, vol. 38, no. 1, pp. 123, 1993.

[15] A Sitek, EVR Di Bella, and GT Gullberg, "Factor analysis with a priori knowledge-application in dynamic cardiac spect," Physics in medicine and biology, vol. 45, no. 9, pp. 2619, 2000.

[16] M Filippi, M Desvignes, E Moisan, C Ghezzi, P Perret, and Fagret D, "A priori spatiaux et analyse factorielle de séquences scintigraphiques," in GRETSI, 2015.

[17] Arkadiusz Sitek, Grant T Gullberg, and Ronald H Huesman, "Correction for ambiguous solutions in factor analysis using a penalized least squares objective," Medical Imaging, IEEE Transactions on, vol. 21, no. 3, pp. 216-225, 2002. 